\title{
Bowel obstruction and pelvic mass
}

\section{Ally Murji MD, Mara L. Sobel MSc MD}

Competing interests: None declared.

This article has been peer reviewed.

Correspondence to: Dr. Mara L. Sobel, mara.sobel@utoronto.ca

CMAJ 2011. DOI:10.1503 /cmaj.091851

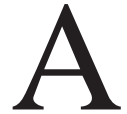
42-year-old woman presented to the emergency department with a four-day history of severe constipation, bilious vomiting and colicky abdominal pain. Her symptoms started with the onset of menses but differed from the crampy dysmenorrhea for which she had taken over-the-counter analgesics for many years. She had no history of bloody or black stool, or changes in her bowel function. Apart from a 20 pack-year smoking history, her medical, surgical and family histories were noncontributory.

On examination, she was afebrile, and her abdomen was soft but distended, with hyperactive, high-pitched bowel sounds. There were no peritoneal signs. With deep palpation, we felt a tender, firm mass in the left lower quadrant. On digital examination, her rectum was empty of stool and contained mucous that tested positive for occult blood. A pelvic examination suggested a retroverted, mobile uterus, with fullness in the left adnexa and tender nodularities in the pouch of Douglas.

Laboratory test results were within normal limits except for a leukocyte count of 16 (normal $3-10) \times 10^{9} / \mathrm{L}$. An abdominal radiograph showed dilated loops of bowel and multiple air-fluid levels, consistent with bowel obstruction. A computed tomography scan confirmed an obstruction in the distal portion of the large bowel secondary to a $7-\mathrm{cm}$ rectosigmoid mass with mild ascites. On transvaginal ultrasonography, the mass appeared to be $6 \mathrm{~cm}$ in diameter and in the left adnexal region, extending posterior to the uterus and in contact with the adjacent bowel. The right ovary appeared normal.

\section{What is the next most appropriate diagnostic test or procedure?}
a. Testing for the cancer antigen 125 (CA-125) tumour marker
b. Magnetic resonance imaging (MRI)
c. Colonoscopy
d. Exploratory laparotomy

Given the findings of bowel obstruction and a rectosigmoid mass in our patient, we suspected colorectal carcinoma. We proceeded with (c) a colonoscopy, with the intent of obtaining a tissue sample for diagnosis. Surprisingly, no mucosal lesions were found. However, at the obstruction site, the mucosa was edematous with an acute angulation of the sigmoid colon, compatible with extraluminal compression. These findings, together with the previous imaging results, pointed to external compression of the bowel from an adnexal mass or an intramural gastrointestinal lesion. As part of the recommended workup for a pelvic or ovarian mass, ${ }^{1}$ we ordered a CA-125 test; the results were not immediately available.

We felt that MRI was the next most appropriate noninvasive diagnostic test. The MRI images showed a mass arising from within the bowel wall and extending to the subserosa (Figure 1). Both ovaries appeared normal.

Our next step was an urgent exploratory laparotomy, which showed a nodular rectosigmoid mass that was adherent to the rectovaginal septum. We mobilized the lesion, resected the diseased bowel and created a diverting colostomy using the Hartmann procedure. A

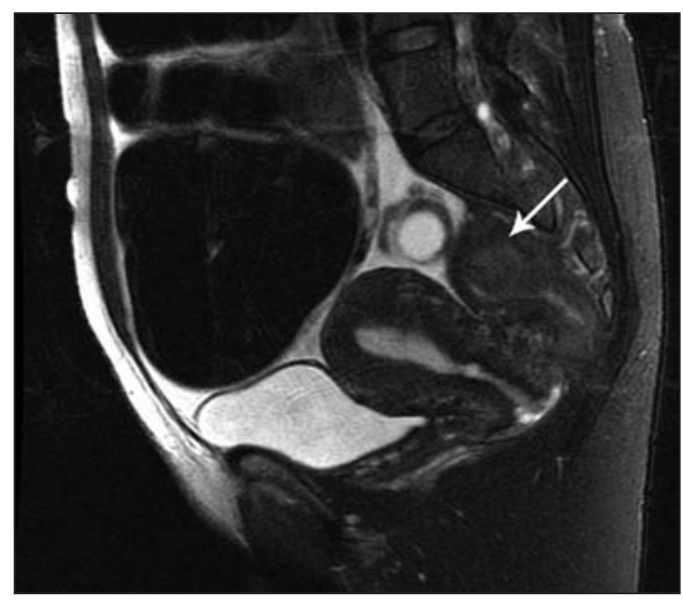

Figure 1: Sagittal $T_{2}$-weighted magnetic resonance image showing a colonic mass (arrow) in a 42year-old woman with symptoms of large-bowel obstruction. 
thorough examination of the pelvis did not find additional areas of disease.

\section{What is your diagnosis?}

a. Gastrointestinal stromal tumour

b. Gastrointestinal lymphoma

c. Sigmoid phlegmon (inflammatory mass)

d. Deeply infiltrating gastrointestinal endometriosis

\section{Discussion}

After reviewing the pathology findings (Figure 2), we diagnosed (d) deeply infiltrating gastrointestinal endometriosis. Foci of endometriosis were also identified in mesenteric lymph nodes (Figure 3).

Endometriosis is characterized by the presence of functional endometrial glands and stroma outside of the uterus. This common condition affects up to $10 \%$ of women of reproductive age, most often involving the ovaries and uterosacral ligaments. Gastrointestinal involvement occurs in up to $34 \%$ of women with pelvic endometriosis. ${ }^{2}$ The diagnosis of gastrointestinal endometriosis may be difficult to establish be cause its symptoms are relatively nonspecific. Patients may present with abdominal pain, bloating, tenesmus, dyschezia (painful bowel movements), rectal bleeding, diarrhea, constipation or obstruction. ${ }^{3}$ The differential diagnosis is often broad; the main considerations include appendicitis, diverticulitis, inflammatory bowel disease, irritable bowel syndrome and malignant disease. The incidence of endometriosis causing bowel obstruction is unknown, although complete obstruction occurs in less than $1 \%$ of patients with gastrointestinal endometriosis. ${ }^{4}$

The symptoms associated with gastrointestinal endometriosis vary according to the depth and site of involvement. Disease of the small intestine usually manifests as abdominal pain, bloating and obstruction, whereas patients with colonic endometriosis are more likely to present with altered bowel habits (decreased stool calibre, diarrhea, constipation, hematochezia) and an abdominal mass. ${ }^{5}$ Early in the disease process, symptoms may be associated with menses, with cyclical episodes present in $40 \%$ of patients with gastrointestinal involvement. ${ }^{6}$ Often, however, symptoms become continuous as the lesions infiltrate the bowel wall and lead to localized fibrosis, smooth muscle hypertrophy and luminal stenosis.

Adding to the challenge of diagnosing gastrointestinal endometriosis is the often falsenegative result of endoscopic biopsies. The biop- sies tend to be superficial and miss the foci of endometriosis that are located in the deeper bowel layers. When deeply infiltrating gastrointestinal endometriosis is suspected clinically, transvaginal ultrasonography and MRI may prove valuable in reaching the diagnosis. With a sensitivity of $91 \%$ and a specificity of $97 \%$, transvaginal ultrasonography is the first-line imaging modality for such patients. ${ }^{7.8}$ Likewise, MRI detects invasive intestinal endometriosis

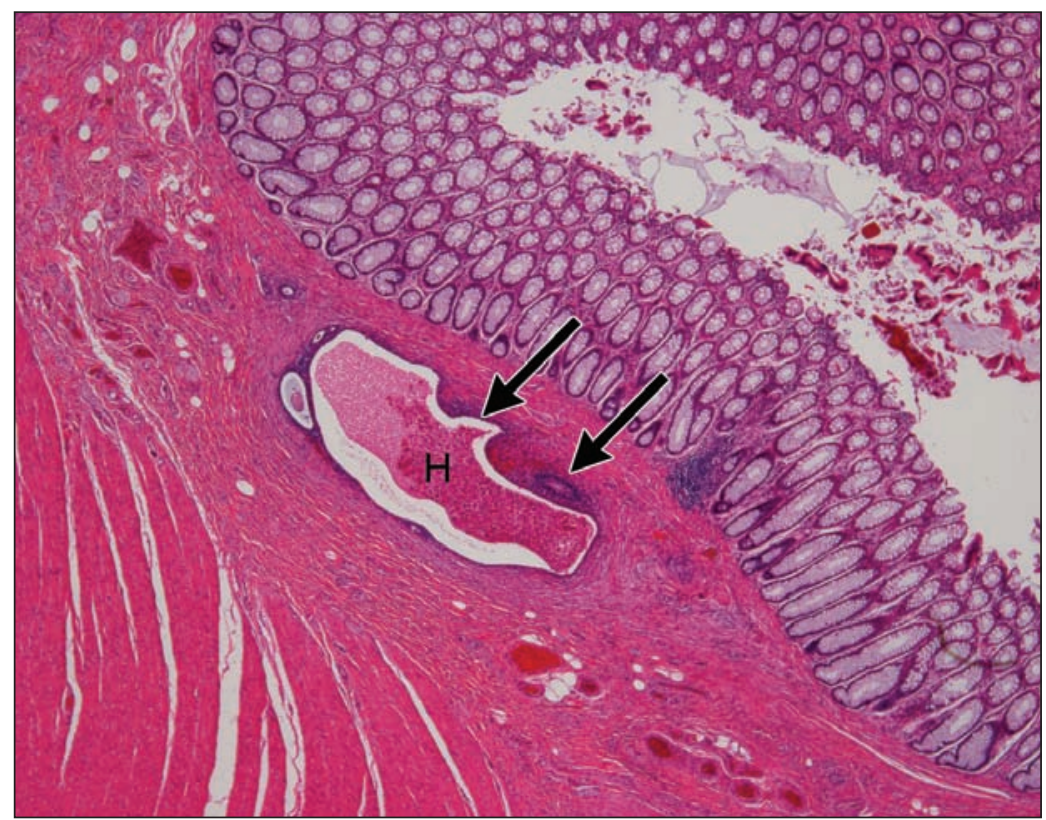

Figure 2: Specimen from colon resection. Endometriotic glands (arrows), stroma and accompanying hemorrhage $(\mathrm{H})$ caused marked thickening of the bowel wall and luminal narrowing (hematoxalin-eosin stain, original magnification $\times 20$ ).

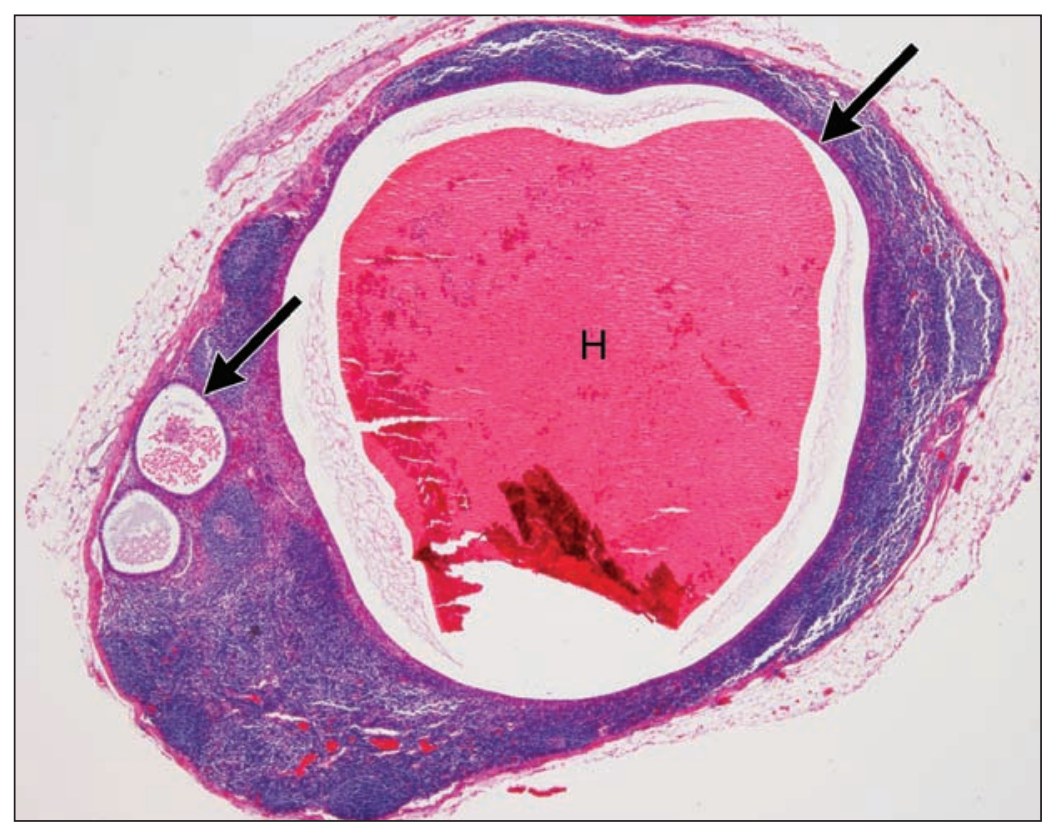

Figure 3: Mesenteric lymph node from colon resection showing foci of endometriosis (arrows) and hemorrhage $(\mathrm{H})$ (hematoxalin-eosin stain, original magnification $\times 20$ ). 
with a sensitivity of $88 \%$ and a specificity of $93 \%$, and it may be useful when the results of ultrasonography are equivocal. ${ }^{9}$ Although CA125 levels may be elevated in endometriosis, the test is primarily used as a tumour marker, with a sensitivity of $80 \%$ for advanced ovarian cancer. However, it has poor specificity and may be elevated in several benign and malignant conditions, including endometriosis and colon cancer. ${ }^{10}$

\section{Colonic masses with obstruction}

A clinical presentation of an abdominal mass and bowel obstruction in the context of worsening gastrointestinal symptoms and equivocal diagnostic tests frequently raises the suspicion of gastrointestinal cancer. Negative findings on colonoscopy do not necessarily rule out malignant disease, because primary extraluminal neoplasms and neoplastic changes arising from endometriosis (endometrioid adenocarcinoma and clear-cell adenocarcinoma) frequently involve only the outermost layers of the colon. The differential diagnosis of intramural gastrointestinal lesions causing large bowel obstruction is shown in Table 1.,11,12 Although primary colorectal lymphoma may also present as an extraluminal mass, mucosal involvement often can be seen with colonoscopy. ${ }^{11}$

The presence of a colonic mass with intact mucosa can also indicate sigmoid phlegmon, the most common complication of diverticular dis- ease. ${ }^{11}$ Patients with acute diverticulitis tend to present with pain in the left lower quadrant of the abdomen, altered bowel habits, low-grade fever and mild leukocytosis. On pelvirectal examination, a tender mass may be palpable. Computed tomography is the diagnostic test of choice for acute diverticulitis. ${ }^{11}$ In our patient, phlegmon was unlikely because of the absence of both colonic diverticula and signs of inflammation of the bowel wall on imaging.

\section{Management}

Endometriosis can be managed both medically and surgically. In its clinical practice guidelines on the treatment of endometriosis, the Society of Obstetricians and Gynaecologists of Canada recommends combined estrogen-progestin hormonal contraceptives or progestin alone as firstline medical therapy. Gonadotropin-releasing hormone agonists and the levonorgestrelreleasing intrauterine device should be considered second-line therapeutic options. Nonsteroidal anti-inflammatory drugs and opioids may provide effective analgesia while awaiting symptom resolution from hormonal therapy. Surgery is generally reserved for refractory symptoms, severe invasive disease, infertility, pelvic mass or diagnostic uncertainty. ${ }^{13}$

Management of gastrointestinal endometriosis depends on the extent of disease. The American Congress of Obstetricians and Gynecologists

\begin{tabular}{|c|c|}
\hline Condition & Characteristics \\
\hline Deeply infiltrating endometriosis & $\begin{array}{l}\text { - Patients present with dysmenorrhea, chronic pelvic pain, deep } \\
\text { dyspareunia and dyschezia } \\
\text { - Symptoms are initially cyclical but may become continuous as } \\
\text { the disease progresses } \\
\text { - Diagnosis can be aided by transvaginal ultrasonography and } \\
\mathrm{MRI}^{8}\end{array}$ \\
\hline $\begin{array}{l}\text { Diverticulitis with sigmoid } \\
\text { phlegmon }\end{array}$ & $\begin{array}{l}\text { - This condition is a complication of acute diverticulitis } \\
\text { - Patients present with pain in the left lower quadrant, altered } \\
\text { bowel habits and low-grade fever } \\
\text { - In the acute phase, CT is often diagnostic, showing an inflamed } \\
\text { soft-tissue mass } 11\end{array}$ \\
\hline $\begin{array}{l}\text { Gastrointestinal mesenchymal } \\
\text { neoplasm* }\end{array}$ & $\begin{array}{l}\text { Patients with this rare type of neoplasm present with nonspecific } \\
\text { gastrointestinal symptoms (bloating, early satiety), bleeding, } \\
\text { pain and symptoms of bowel obstruction } \\
\text { - CT is the imaging modality of choice, showing a smoothly } \\
\text { contoured mass }{ }^{11}\end{array}$ \\
\hline Colorectal lymphoma & $\begin{array}{l}\text { - Patients present with pain, weight loss and symptoms of an } \\
\text { abdominal mass } \\
\text { - Colonoscopy is often diagnostic, showing diffuse mucosal } \\
\text { nodularity, induration, ulceration and a mass }{ }^{11}\end{array}$ \\
\hline
\end{tabular}


recommend suppression of ovarian function with a gonadotropin-releasing hormone agonist as first-line medical treatment of extrapelvic endometriosis. For patients presenting with bowel obstruction or those with severely advanced intestinal disease, surgical resection is the treatment of choice. ${ }^{7}$ Although there is no evidence supporting the use of preoperative medical suppressive therapy, a postoperative course of hormonal therapy (three months or longer) is often prescribed for patients with residual disease or persistent pain, or to extend the pain-free interval after surgery. ${ }^{7}$ We prescribed a gonadotropinreleasing hormone agonist for our patient. At the last follow-up visit, she was awaiting the reversal of her colostomy.

\section{References}

1. Le T, Giede C, Salem S, et al. Initial evaluation and referral guidelines for the management of pelvic/ovarian masses. J Obstet Gynaecol Can 2009;31:668-73.

2. Kratzer GL, Salvati EP. Collective review of endometriosis of the colon. Am J Surg 1955;90:866-9.

3. De Ceglie A, Bilardi C, Blanchi S, et al. Acute small bowel obstruction caused by endometriosis: a case report and review of the literature. World J Gastroenterol 2008;14:3430-4.

4. de Bree E, Schoretsanitis G, Melissas J, et al. Acute intestinal obstruction caused by endometriosis mimicking sigmoid carcinoma. Acta Gastroenterol Belg 1998;61:376-8.

5. Yantiss RK, Clement PB, Young RH. Endometriosis of the intestinal tract: a study of 44 cases of a disease that may cause diverse challenges in clinical and pathologic evaluation. Am J Surg Pathol 2001;25:445-54.

6. Jubanyik KJ, Comite F. Extrapelvic endometriosis. Obstet Gynecol Clin North Am 1997;24:411-40.

7. Practice bulletin no. 114: management of endometriosis. Obstet Gynecol 2010;116:223-36.

8. Piketty M, Choplin N, Dousset B, et al. Preoperative work-up for patients with deeply infiltrating endometriosis: transvaginal ultrasonography must definitely be the first-line imaging exami- nation. Hum Reprod 2009;24:602-7.

9. Bazot M, Bornier C, Dubernard G, et al. Accuracy of magnetic resonance imaging and rectal endoscopic sonography for the prediction of location of deep pelvic endometriosis. Hum Reprod 2007;22:1457-63.

10. Clarke-Pearson DL. Screening for ovarian cancer. $N$ Engl J Med 2009;361:170-7.

11. Corman ML, editor. Colon and rectal surgery. 5th ed. Philadelphia (PA): Lippincott Williams \& Wilkins; 2005.

12. Yantiss RK, Clement PB, Young RH. Neoplastic and pre-neoplastic changes in gastrointestinal endometriosis: a study of 17 cases. Am J Surg Pathol 2000;24:513-24.

13. Leyland NA, Casper R, Laberge P, et al. Society of Obstetricians and Gynaecologists of Canada clinical practice guideline. Endometriosis: diagnosis and management. J Obstet Gynaecol Can 2010;7(2 Suppl):S1-S32.

Affiliations: From the Department of Obstetrics and Gynecology, University of Toronto, Toronto, Ont.

Contributors: Both authors contributed substantially to writing the manuscript and revising it for important intellectual content and approved the final version submitted for publication.

Acknowledgements: The authors thank Dr. Maja Barnard for help with the histological images and Dr. Caroline Chan and Mr. Ashish Papneja for help with the clinical images, details of the case and the initial literature review. The authors also thank these people, and Dr. Paul Shuen, the clinical physician who supervised the case, for their help in reviewing the manuscript.

\section{Resources}

- Society of Obstetricians and Gynaecologists of Canada: Clinical practice guidelines on the diagnosis and management of endometriosis (www.sogc.org/guidelines/documents /gui244CPG1007E.pdf)

- Practice bulletin no. 114: management of endometriosis. Obstet Gynecol 2010;116:223-36. Summary available through the National Guideline Clearinghouse at www.guideline.gov.

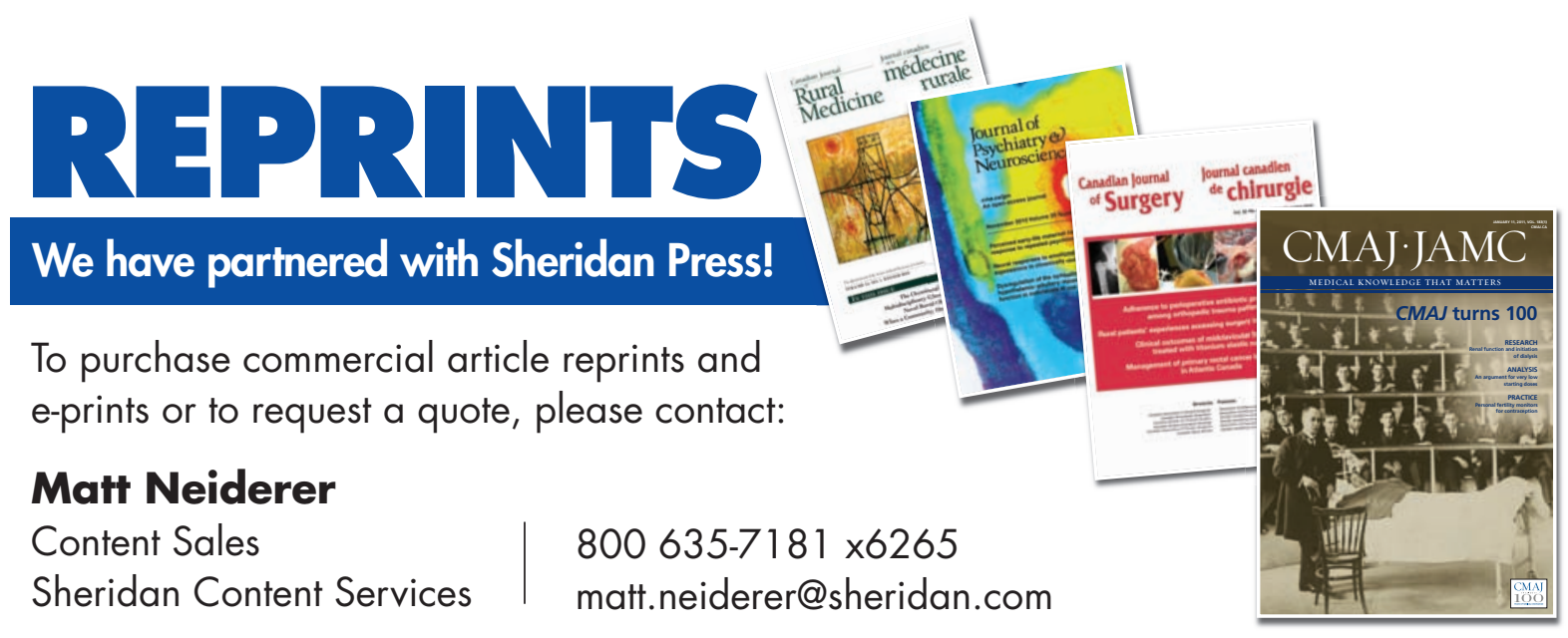

